\title{
Bulk and surface plasmons in highly nanoporous gold films
}

\author{
A I Maaroof, A Gentle, G B Smith*, and M B Cortie \\ Institute for Nanoscale Technology, University of Technology, Sydney \\ PO Box 123, Broadway NSW 2007, Australia \\ *Corresponding author: Geoff.Smith@uts.edu.au (Geoff B. Smith) \\ PACS code: 78.20.Ci, 78.55.Mb, 78.66.-W, 78.67.-n, 68.55.-a
}

\begin{abstract}
The far-field plasmonic behaviour of nanoporous gold films with void densities, $f$, ranging from $60 \%$ to $90 \%$ has been investigated and modelled. These layers have good dc conductivity and quite different nanostructure to traditional porous layers in which the metal percolates. Our gold films with $f$ above $70 \%$ have high thermal emittance for a conductor at their thicknesses, and their flat spectral response at visible and near infrared wavelengths is not metal-like. We derive effective optical constants which become plasmonic at wavelengths between $1.8 \mu \mathrm{m}$ and $4 \mu \mathrm{m}$ for $f$ from $72 \%$ to $87 \%$. This onset is at much longer wavelengths than that in bulk gold. For $f$ below 70\% the onset of plasmonic behaviour is much closer to the dense material. A simple test is implemented to test for surface plasmon polaritons (SPP) under illumination. The more porous films show no evidence of SPP, while the less porous films display weak evidence. Thus by tailoring $f$ in these nanostructures we can tailor the onset of effective plasmonic response across a wide range and emittance from around 0.9 down to low values. An effective uniform metal response is thus found in the presence of surface nanostructure without the interface absorption found in dense gold layers with structured surfaces.
\end{abstract}

\section{Introduction}

Most optical properties reported to date on thin conducting films containing voids or pores display strong departures from normal metal behaviour [1-4] at near infrared (NIR) wavelengths, and for some metals, at visible wavelengths. A variety of complex responses have been reported including resonant absorption accompanied by strong, random local fields $[1,3]$, and anomalously high transmittance for certain wavelengths for nano-size pores [5,6]. Optical anomalies in granular metal films of noble metals, where the metal percolates but voids remain, have been known for some time, and could not be explained satisfactorily using basic effective medium models [7]. In these structures, and in most cases where optical anomalies have been reported in percolating layers, the voids traverse the film from one side to the other. Explanations of optical response in these layers require models which go beyond bulk effects in the composite to include interface effects, which arise because such structures allow surface plasmon polaritons (SPP) to develop at both sides of the metal. SPPs are charge density waves, which are localised at a surface and carry evanescent fields which decay into the metal and adjacent dielectric.

When voids are not present SPPs can still arise due to surface nanostructure or roughness on an otherwise dense layer [8]. These may lead to undesirable optical effects, in particular enhanced losses, which were not allowed for in the thin film design process. In such cases spectral properties may also have obvious resonance based features. Some nanostructures on metal films however may yield a wide spectrum of resonances [2,9] which in the far fields combine to give what appears at first sight to be an average metal-like or Drude response. However detailed analysis and recent experiments $[10,11]$ show that additional absorption will occur at the interface due to the excitation of SPPs, and thus that standard thin film models cannot fully describe the optical properties of such films. One approach to include this effect in standard models is to introduce an additional very thin layer at the interface to obtain 
a fit [11]. While such a "non-physical" layer may yield a fit to optical data by "allowing" more light to enter the layer and be absorbed, there is no a priori way of establishing its properties without an understanding of the link between surface nanostructure and SPP characteristics. Additional confusion arises because many authors treat surface nanoroughness via an effective medium or optically homogenized interface layer, which is "physical". (Does one then add a further layer in this model to allow for SPP?) Again fits will be possible by adjusting say void density or index gradient, but actual interface structure is rarely used in such models and SPPs are typically absent. Producing very thin metal layers, which are free of such interface effects, is quite challenging. Alternately one can choose to live with the intrinsic surface structure and apply an understanding to modify the design of film stacks so as to damp such effects to acceptable levels. Metal layers are of increasing technological importance in areas such as solar control glazing, and mirrors [12,13], subwavelength optical imaging [14], spectroscopy, the "super lens" [15], and in thermal control via emittance control. Thicknesses of 10 to $15 \mathrm{~nm}$ are often needed. In most of these low loss, and hence low interface absorption, is important. The ability to tailor plasmonic response is also of growing technical interest.

Surface plasmons can be localised, as for example on the surfaces of nanoparticles or voids. In this case the associated absorption resonances show up in optical spectra as narrow band features. Structural statistics can also lead to averaging over many different localised resonances, with the result being a broader, less distinct band. Once macroscopic conduction or percolation occurs, then delocalised surface plasmons or SPP can also arise. These are expected to add spectral features associated with characteristic length scales on a nanostructured surface.

The films discussed in this paper have the following attributes, which help address one or more of these issues :

(i) Metal-containing layers with good conducting properties and surface nanostructure, and weak or absent SPPs under illumination.

(ii) Highly nanoporous metal films, free of those optical anomalies found in granular and related nanoporous metal layers.

(iii) Metal-insulator composites, which behave rigorously like a conducting metal with a tuneable plasmonic frequency $\left(\omega_{\mathrm{p}}\right)$ at long wavelengths.

(iv) Thermal emittance (e) of electrically conducting layers, which can be controlled over a wide range.

We extend an earlier study of nanoporous conducting gold layers [16,17] to address their surface and bulk plasmonic properties and highlight the contrast in their behaviour to other classes of porous metal layers studied to date. Bulk plasmonic response is analysed in terms of experimental optical data, effective medium models and effective Drude parameters. A simple test to assess if intrinsic SPPs are present under illumination is also established and used. We will then demonstrate for these structures that it is possible to combine good electrical conductivity with metal-like optical response without having to be concerned with surface plasmon polaritons, even though surface nanostructure is quite obvious. As well as extending the data on earlier samples to SPP effects and emittance, new samples with different void content are added into this study to extend the scope of properties reported. Void content is easily controlled via deposition conditions and use of different thicknesses. The breadth of effective plasmonic tuning that is possible in mesoporous metals in which the void structure is a complex 3-dimensional nanoscale network, rather than a series of connecting channels between film surfaces, is wide. We shall show that with techniques for making such structures now well established and reproducible, varying void content and nanostructure to shift effective plasmonic response and associated properties is now quite straightforward. 


\section{Experimental details}

\subsection{Porous Film Fabrication}

The fabrication process of the mesoporous gold films is similar to that described previously $[16,17]$. Briefly, alloy films of $\mathrm{AuAl}_{2}$ were prepared by co-depositing the elements using high vacuum dc magnetron sputtering onto glass substrates. The sputtering targets of $\mathrm{Au}$ and $\mathrm{Al}$ were $99.999 \%$ pure discs $(50 \mathrm{~mm}$ diameter), placed $150 \mathrm{~mm}$ away from the substrate. The base pressure was better than $\sim 10^{-6}$ Torr, while sputtering was carried out in the presence of flowing $\mathrm{Ar}$ at an inlet gas pressure of 8mTorr. Varying pressure enabled a wider variety of void content to be produced [18]. To ensure good homogeneity, uniformity and crystallinity, a rotating stage at $400^{\circ} \mathrm{C}$ substrate temperature was used during deposition. All the films were produced at different thicknesses using a crystal monitor. After co-depositing of $\mathrm{AuAl}_{2}$ on a glass substrate, aluminium was removed from the compound by immersing the films in $\mathrm{NaOH}(0.2 \mathrm{M})$ solution. Films with the highest effective plasma frequencies and lowest thermal emittance were made from alloys deposited with ion assistance, figure 1(d). Those with lowest $\left(\omega_{\mathrm{p}}\right)$ were deposited without ion assistance, figure 1(a-c).

\subsection{Characterisation}

2.2.1. Microscopy. Scanning electron microscopy (SEM) on a Zeiss Supra 55VP was used to study the nanostructure and cross sections of the films. All films for imaging were deposited at different thicknesses onto $4 \mathrm{~nm}$ carbon on a glass substrate. The thin carbon layer was added to the glass for imaging purposes. We have verified that this layer has a negligible effect on the optical and morphological properties of the mesoporous gold films.

2.2.2. Spectrophotometry. Optical spectral measurements of transmittance $T(\lambda)$ and reflectance $R(\lambda)$ were carried out using a Perkin-Elmer Lambda 950 UV/VIS/NIR spectrophotometer over the visible and near-infrared wavelength range $2500 \mathrm{~nm} \geq \lambda \geq 300 \mathrm{~nm}$ on all mesoporous gold films.

2.2.3. Ellipsometry. A UVISEL spectroscopic phase modulated ellipsometer was used to measure the changes in phase $(\psi)$ and amplitude $(\Delta)$ of the electromagnetic waves incident on the films after reflection. Three different angles of incident, $60^{\circ}, 65^{\circ}$, and $70^{\circ}$ were used and the spectral range was $800 \mathrm{~nm} \geq \lambda \geq 300 \mathrm{~nm}$. Combining ellipsometry data with transmittance data allowed the optical thickness of the films to be determined with a high degree of accuracy.

2.2.3. Emissometer. Thermal emittance of the mesoporous gold films was measured using a Windbourn rotating dual cavity emissometer, in which the cavity irradiating the sample is heated to about $100^{\circ} \mathrm{C}$. The proportion of this radiation reflected to the compact thermopile detector is sampled and fed to a lock-in amplifier. A copper plate was scanned as the reference sample. Reflectance values of each sample and of the standard were used to calculate $R$ and hence the values of the emittance using Kirchhoff's law $(E=1-R)$.

\section{Results and discussion}

\subsection{Nanostructure of mesoporous gold films}

Figure 1(a,b and c) show typical SEM images of mesoporous gold films for which the alloy was deposited without ion assistance. Figure 1(d) is an example of a film deposited with ion assistance. (Further images of films of the type shown in figure 1(d) can be found in reference [18].) The first three images demonstrate that void structure does vary with layer thickness for the same deposition conditions. The main difference shown in figure 1(d) is that the latter has less surface nanostructure and is denser overall. A smooth, dense gold layer was deposited 
under the same conditions as the alloys. It was used both as an optical and structural reference. A micrograph showing its grain structure is given in figure 1(e).

As film thickness increases for layers derived from alloy films deposited without ion assistance, it can be seen that larger, inter-connected units of gold develop. These units themselves are porous with many nanovoids around $5 \mathrm{~nm}$ across. These larger units are typically $50 \mathrm{~nm}$ to $150 \mathrm{~nm}$ across, are separated by about 25 to $50 \mathrm{~nm}$, and interconnected with finer gold strands. In contrast the layers deposited with ion assistance do not develop these larger gold units and thus appear more mesh-like in appearance.

\subsection{Optical response of mesoporous gold films}

The results of the optical response measurements of specular transmittance $T(\lambda)$ and reflectance $R(\lambda)$ of the samples illustrated in figures $1(a-c)$ are given in figures 2 (a) and (b) respectively. The films with higher effective plasma frequency [18] (as in figure 1(d)) have qualitatively different spectral response at these wavelengths, with their plots looking more like those shown for void-free gold, but shifted slightly to longer wavelengths. All samples are effectively plasmonic, as we will show later, though at first sight the plots in Figures 2 seem to be for non-metallic material (a comment made in our earlier work on these layers [16] which we now qualify in this paper). The basic reason is that the wavelength location of inter-band transitions is intrinsic to the metal present and does not change significantly as void content changes, but in contrast effective $\omega_{\mathrm{p}}$ values shift over a wide range. Both parameters influence the actual frequency below which the material becomes plasmonic, that is, where $R l[\varepsilon(\lambda)]$ goes negative. This is often called the shielded plasma frequency $\omega_{\mathrm{p}}{ }^{*}$, and it is lower than $\omega_{\mathrm{p}}$. The rate and extent to which reflectance rises as frequency drops below $\omega_{\mathrm{p}}$ in a conductor depends on the difference between $\omega_{\mathrm{p}}$ and the relaxation frequency $\omega_{\tau}$. In dense gold $\omega_{p} \gg \omega_{\tau}$ but in the films in figure 2 effective $\omega_{p} *$ is approaching $\omega_{\tau}$. This link is shown explicitly in the next section after these parameters are derived for a wide range of porous films. The more metal-like mesoporous films in the near infrared (NIR) have an $\omega_{\mathrm{p}}{ }^{*}$ which remains well above $\omega_{\tau}$. All the mesoporous gold films we analyse are transparent, so optical techniques confidently can be used to determine their thicknesses, which is very important for modelling. It is necessary for such accuracy that incident light reaches the lower interface, which is not possible for opaque films. We used spectroscopic ellipsometry to obtain the film thicknesses as described in reference [16].
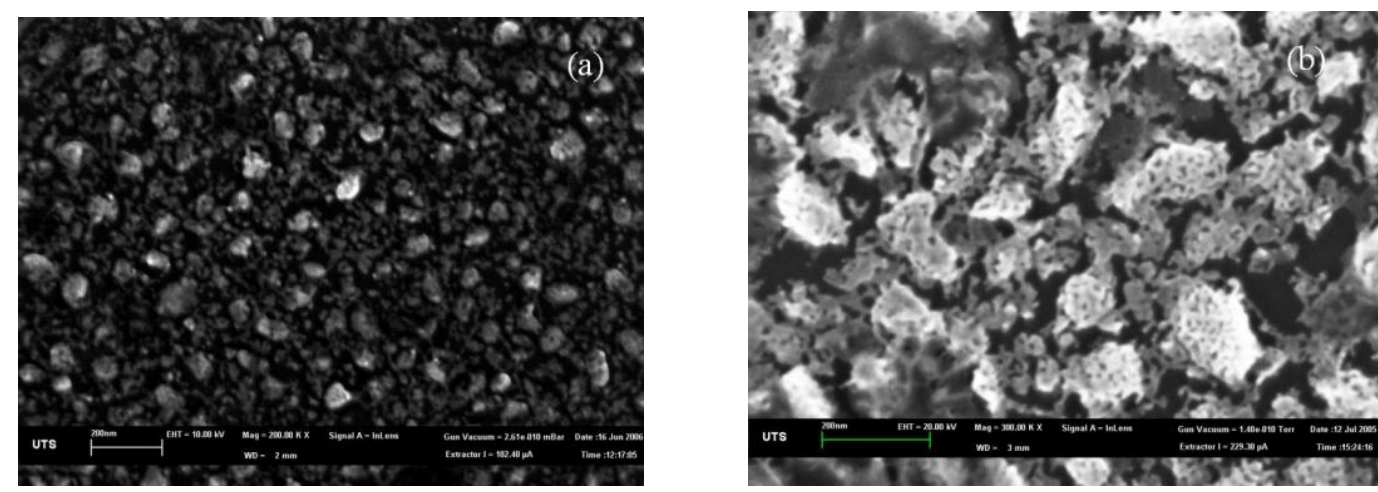

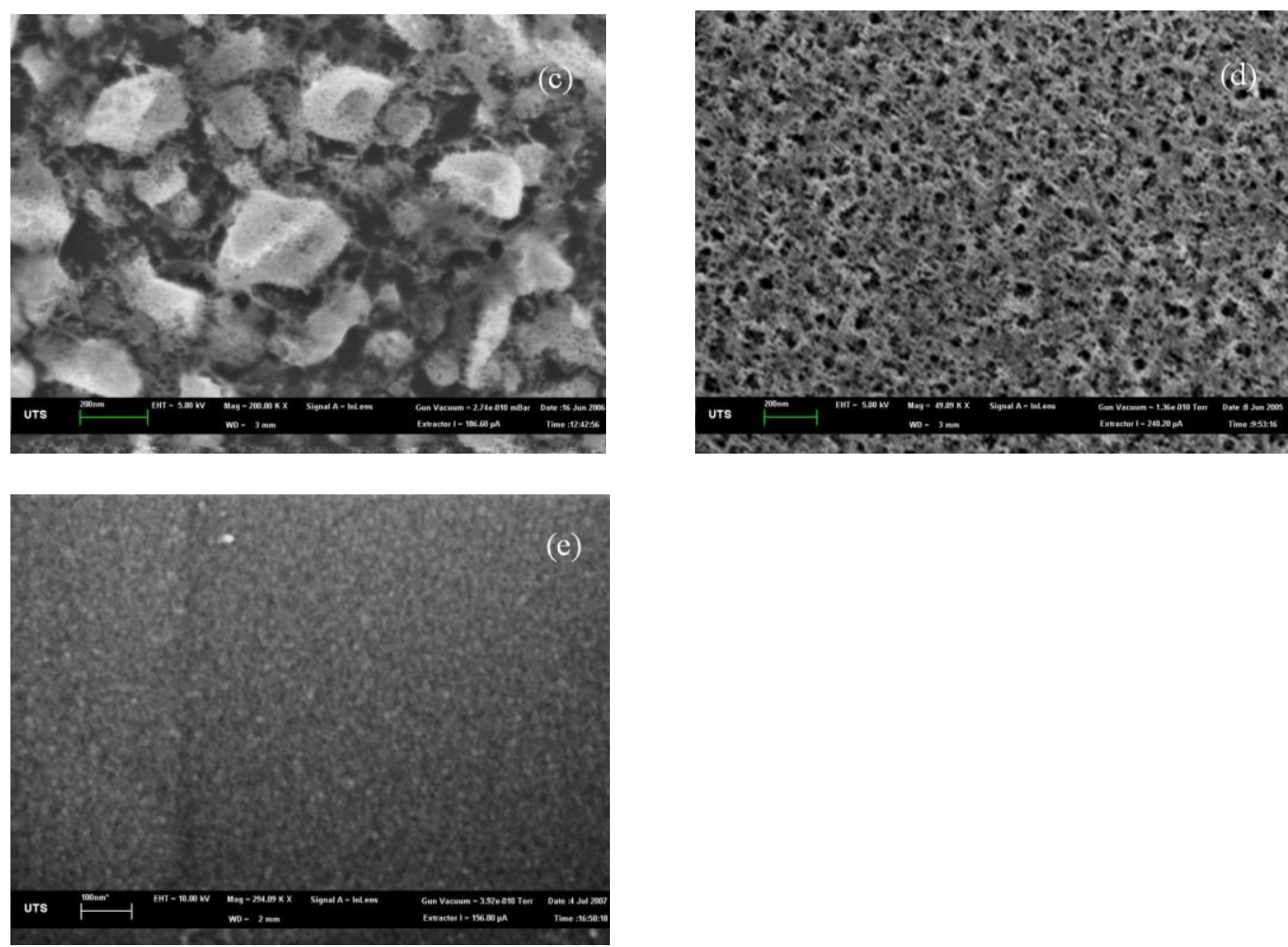

Figure 1. SEM image of mesoporous gold film deposited at $8 \mathrm{mTorr}$ with no ion assistance, final thicknesses (a) $21.95 \mathrm{~nm}$ (b) $37.5 \mathrm{~nm}$ (c) $96.3 \mathrm{~nm}$ in comparison with ion assist (d) 34 $\mathrm{nm}$ and dense gold film (e) $10.7 \mathrm{~nm}$. The scale bars in figures (a-d) are 200nm long and in figure (e) is $100 \mathrm{~nm}$ long.

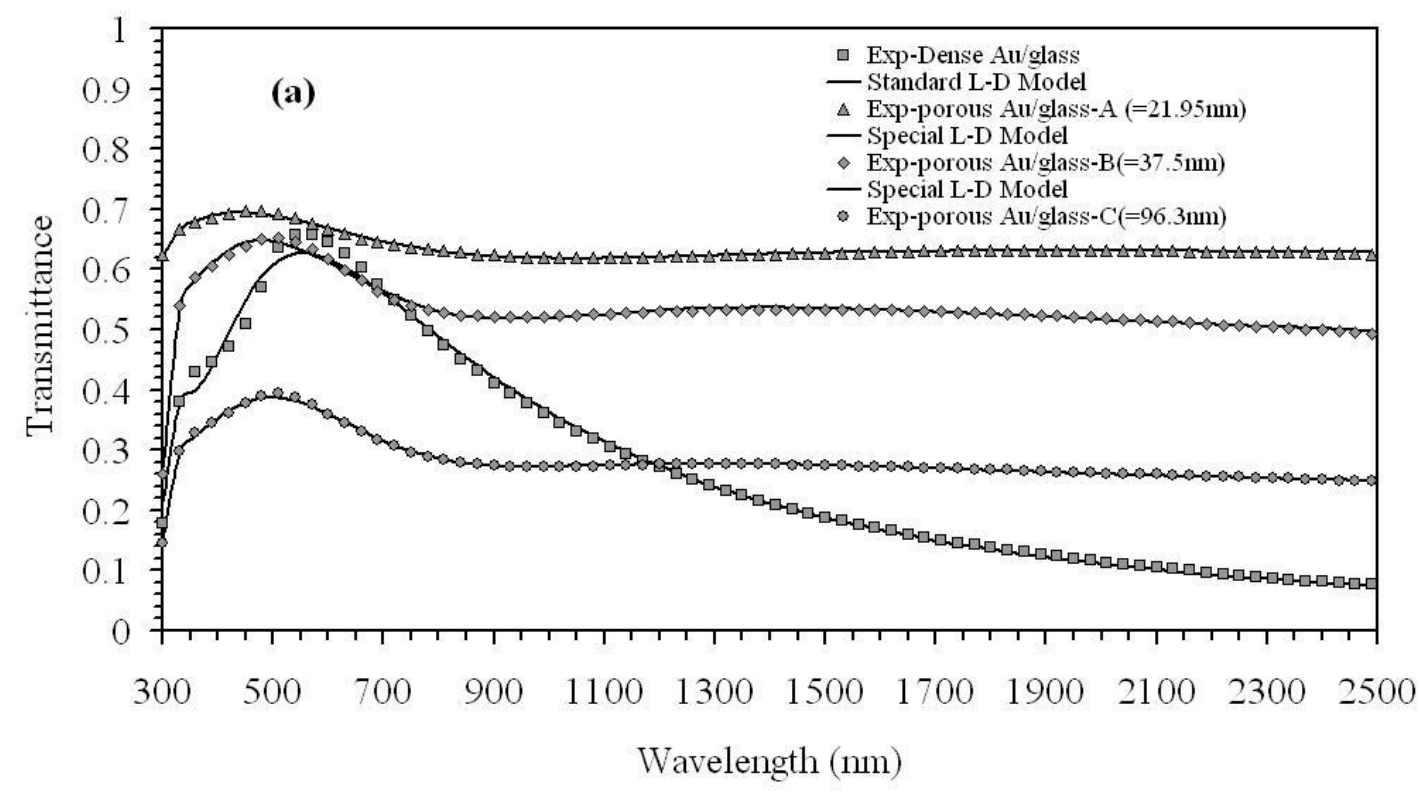




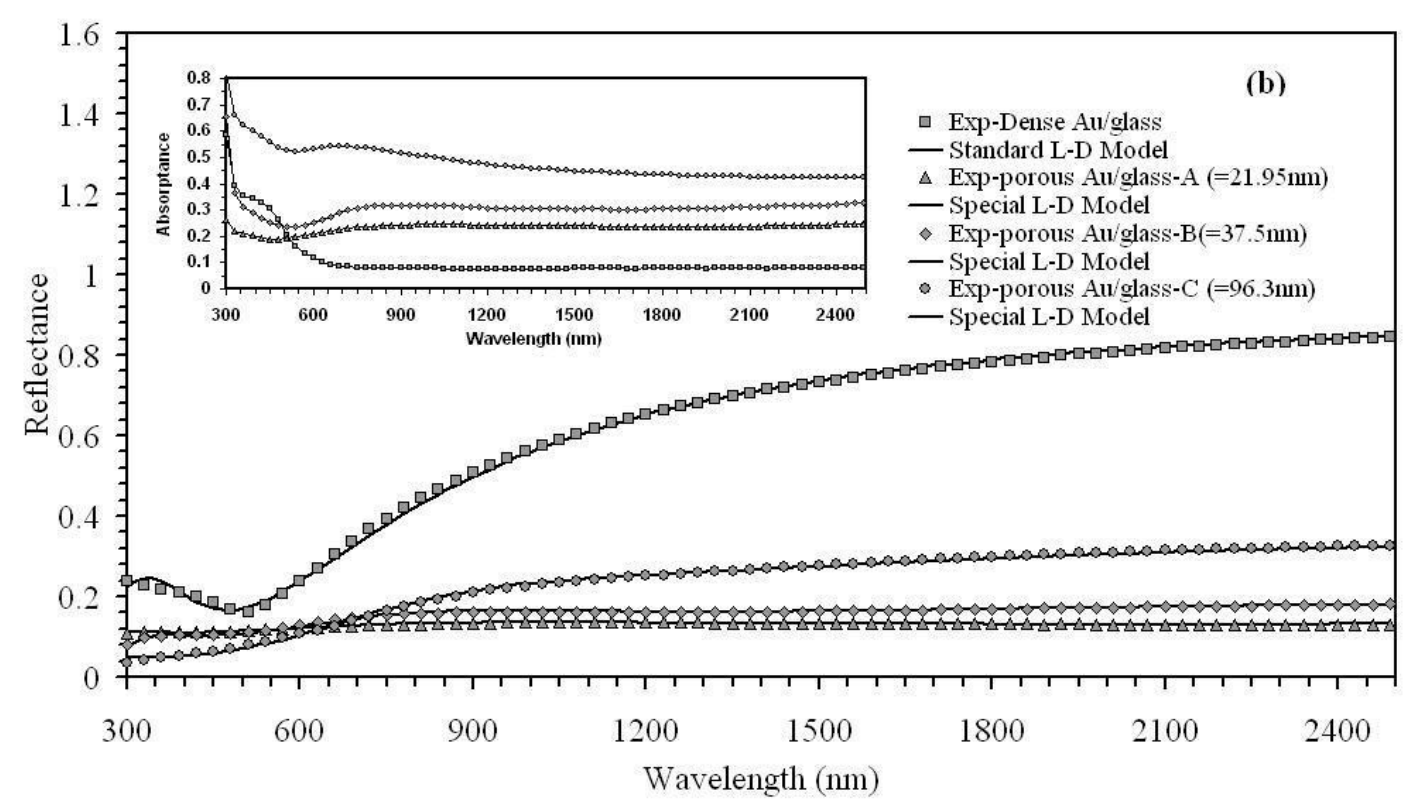

Figure 2. Experimental data for (a) spectral transmittance and (b) spectral reflectance at near normal incidence for three mesoporous gold films. Solid lines represent the fitted models (special L-D model for the mesoporous films and standard L-D model for the $10.7 \mathrm{~nm}$ dense gold film). The inset graph shows absorptance deduced from $A=1-R-T$.

\subsection{Spectral response: theoretical approach}

3.3.1. A homogeneous Lorentz-Drude model for the inhomogeneous layer. Fully dense gold films of order $10 \mathrm{~nm}$ thick or more exhibit a rise in reflection once the film material has a negative dielectric constant, or in other words has a plasmonic optical response. The result for gold is its yellow colour as it becomes plasmonic near $520 \mathrm{~nm}$, followed by a strong infra-red reflectance and very low thermal emittance. In contrast mesoporous gold films do not become plasmonic at visible wavelengths, but rather in the near IR and even well beyond $2.5 \mu \mathrm{m}$ depending on void content, so they are not coloured. The example reflectance measurements in figure 2(b) show that they are all neutral grey in colour. However all do have a rise in reflectance and become plasmonic for $\lambda$ beyond a wavelength threshold which depends on void content and structure. The penalty for a lower plasma frequency is, as we now show in detail, a reduced asymptotic IR reflectance and higher emittance. To understand how the plasmonic response shifts as film structure changes we examine the effective frequencydependent dielectric function $\varepsilon(\omega)$ of the mesoporous gold films. $\varepsilon(\omega)$ represents the homogenised response and data can be fitted with a Lorentz-Drude model $[18,19]$

$$
\hat{\varepsilon}(\omega) \equiv \varepsilon_{1}+\mathrm{i} \varepsilon_{2}=\varepsilon_{\infty}+\sum_{\mathrm{k}} \frac{\mathrm{C}_{\mathrm{k}}}{\omega_{\mathrm{k}}^{2}-\omega\left(\omega+\mathrm{i} \omega_{\tau, \mathrm{k}}\right)},
$$

where $\varepsilon_{\infty}$ is a constant, above 1 , due to the tails of the interband (ib) transitions in the UV, $C_{k}$ is the amplitude of oscillator $\mathrm{k}$ (or its spectral weight), $\omega_{\mathrm{k}}$ is the centre energy, and, $\omega_{\tau, \mathrm{K}}$ is the broadening of each oscillator.

We need only two oscillators for the $10.7 \mathrm{~nm}$ thick dense gold film because of the influence of the $\mathrm{k}=1$ interband transitions in the visible region. The contribution of the free electrons is through the Drude model term, where $\mathrm{k}=0$ and $\omega_{\mathrm{o}}=0$. Therefore the dielectric functions of the dense gold metal can be written for the range of interest as 


$$
\hat{\varepsilon}(\omega) \equiv \varepsilon_{\infty}+\varepsilon(\omega)_{\mathrm{ib}}+\varepsilon(\omega)_{\text {Drude }}
$$

Where $\varepsilon(\omega)_{\mathrm{ib}}$ represents the inter-band part due to $d$ electrons. This term impacts on the transition to a plasmonic regime. $\varepsilon(\omega)_{\text {Drude }}$ represents the Drude part due to free $s$-electrons. Mesoporous films which show a rise in reflectance in the NIR (ie at $\lambda<2.5 \mu \mathrm{m}$ ) and are hence more metal-like can also be fitted at visible and IR wavelengths with the standard 2oscillator model of equation (2). The LD parameters for dense gold and an example of these metal-like porous layer are provided in table 1, along with those of the more porous, less metal-like layers.

The latter mesoporous gold films as in Fig.2, have a peculiar flat spectral response in the NIR and could only be fitted well using this approach by inclusion of a third oscillator located in the near-infrared (NIR) region [20]. The resulting model becomes.

$$
\begin{gathered}
\hat{\varepsilon}(\omega) \equiv \varepsilon_{\infty}+\varepsilon(\omega)_{\mathrm{ib}}+\varepsilon(\omega)_{\mathrm{NIR}}+\varepsilon(\omega)_{\text {Drude }} \\
\hat{\varepsilon}(\omega)=\varepsilon_{\infty}+\left[\frac{\mathrm{C}_{\mathrm{ib}}}{\omega_{\mathrm{ib}}^{2}-\omega\left(\omega+\mathrm{i} \omega_{\tau, \mathrm{ib}}\right)}\right]+\left[\frac{\mathrm{C}_{\mathrm{NIR}}}{\omega_{\mathrm{NIR}}^{2}-\omega\left(\omega+\mathrm{i} \omega_{\tau, \mathrm{NIR}}\right)}\right]-\left[\frac{\omega_{\mathrm{p}}^{2}}{\omega\left(\omega+\mathrm{i} \omega_{\tau}\right)}\right]
\end{gathered}
$$

The origin of this extra term is as yet not understood, but its inclusion enables excellent fits to this class of film as seen in Figures 2(a) and (b) while the two oscillator model does not even work qualitatively [20]. The parameters deduced from fitting with this model are included in table 1 for the three films of figure 1, each with a quite distinct thickness.

\begin{tabular}{|c|c|c|c|c|c|}
\hline $\begin{array}{l}\text { L-D oscillator D } \\
\text { parameter }\end{array}$ & $\begin{array}{c}\text { Dense } \mathrm{Au} \quad \mathrm{F} \\
(10.7 \mathrm{~nm})\end{array}$ & $\begin{array}{rr}\text { Porous Au-A } & \text { Porous Au-B } \\
(21.95 \mathrm{~nm}) & (37.5 \mathrm{~nm})\end{array}$ & $\begin{array}{l}\text { Porous Au-C } \\
(96.3 \mathrm{~nm})\end{array}$ & $\begin{array}{l}\text { Porou } \\
\text { with } \\
\text { assist }\end{array}$ & $\begin{array}{l}\mathrm{Au} \\
4 \mathrm{~nm})\end{array}$ \\
\hline$\varepsilon_{\infty}$ & 4.6 & 1.7 & 2.0 & 2.8 & 1.9 \\
\hline$\omega_{\mathrm{p}}(\mathrm{eV})$ & 8.6 & 2.1 & 2.2 & 2.4 & 4.5 \\
\hline$\omega_{\tau, \text { meso }}(\mathrm{eV})$ & 0.09 & 0.80 & 0.86 & 1.05 & 0.03 \\
\hline $\mathrm{C}_{\mathrm{NIR}}\left(\mathrm{eV}^{2}\right)$ & absent & 6.2 & 3.3 & 2.6 & absent \\
\hline$\omega_{\tau, \mathrm{NIR}}(\mathrm{eV})$ & absent & 2.0 & 1.3 & 1.4 & absent \\
\hline$\omega_{\mathrm{NIR}}(\mathrm{eV})$ & absent & 1.4 & 1.4 & 1.4 & absent \\
\hline $\mathrm{C}_{\mathrm{Au}}\left(\mathrm{eV}^{2}\right)$ & 41.0 & 7.9 & 7.1 & 8.8 & 25 \\
\hline$\omega_{\tau, \text { Au.ib }}(\mathrm{eV})$ & 1.9 & 2.8 & 2.4 & 3.2 & 2.8 \\
\hline$\omega_{\text {Au.ib }}(\mathrm{eV})$ & 3.5 & 3.9 & 3.9 & 3.8 & 3.6 \\
\hline
\end{tabular}

Table 1: Fitted parameters for L-D model including an NIR oscillator for the three different thickness mesoporous gold films without ion assist and one sample with ion assist.

To better grasp the impacts of the variations in Drude parameters, and of the NIR oscillator when present, it is useful to compare the full dispersion relation for $\varepsilon(\omega)$ between bulk gold 
and the various meso-films. This is done in figures 3(a) and 3(b) with the inset plots for three different metal-like meso-gold layers and the main plots for the samples shown in figures 1(ac) and for the dense gold film. Of note is the variation of the onset of plasmonics or $\omega_{\mathrm{p}}{ }^{*}$ (where $\varepsilon_{1}=0$ ), the different curve shapes at longer wavelengths compared with bulk gold, and the qualitative differences between the two types of meso-films (i.e. those with an NIR oscillator and those without). All meso-gold films become plasmonic, but only weakly so compared with dense gold because their IR response quickly asymptotes by around 6 to 10 $\mu \mathrm{m}$. This cannot be attributed to the fixed inter-band term, plus the drop in $\omega_{\mathrm{p}}$ with void density combining with the rise in $\omega_{\tau}$. When the void density reaches values at which the NIR oscillator is significant, its effect dominates because it shifts the onset of plasmonic response $\left(\omega_{p}^{*}\right)$ to even lower wavelengths than it would be without this new oscillator. This in turn exacerbates the impact of $\omega_{\tau}$ and leads to a rapid approach to a quite weak asymptotic negative value of $\varepsilon_{1}$. The point of inflection in the $\varepsilon_{1}$ plot between 900 and $2400 \mathrm{~nm}$, depends on the sample, and is a good indicator that relaxation rate is starting to dominate. This inflection point does not occur in the dense gold plot here, as it occurs at much longer wavelengths than $6 \mu \mathrm{m}$. The additional oscillator for the more porous layers pushes $\omega_{\mathrm{p}}{ }^{*}$ out to between $1.8 \mu \mathrm{m}$ to $3.6 \mu \mathrm{m}$. It also introduces structure into these $\varepsilon_{1}$ and $\varepsilon_{2}$ curves not found for the meso-layers with lower hole densities.

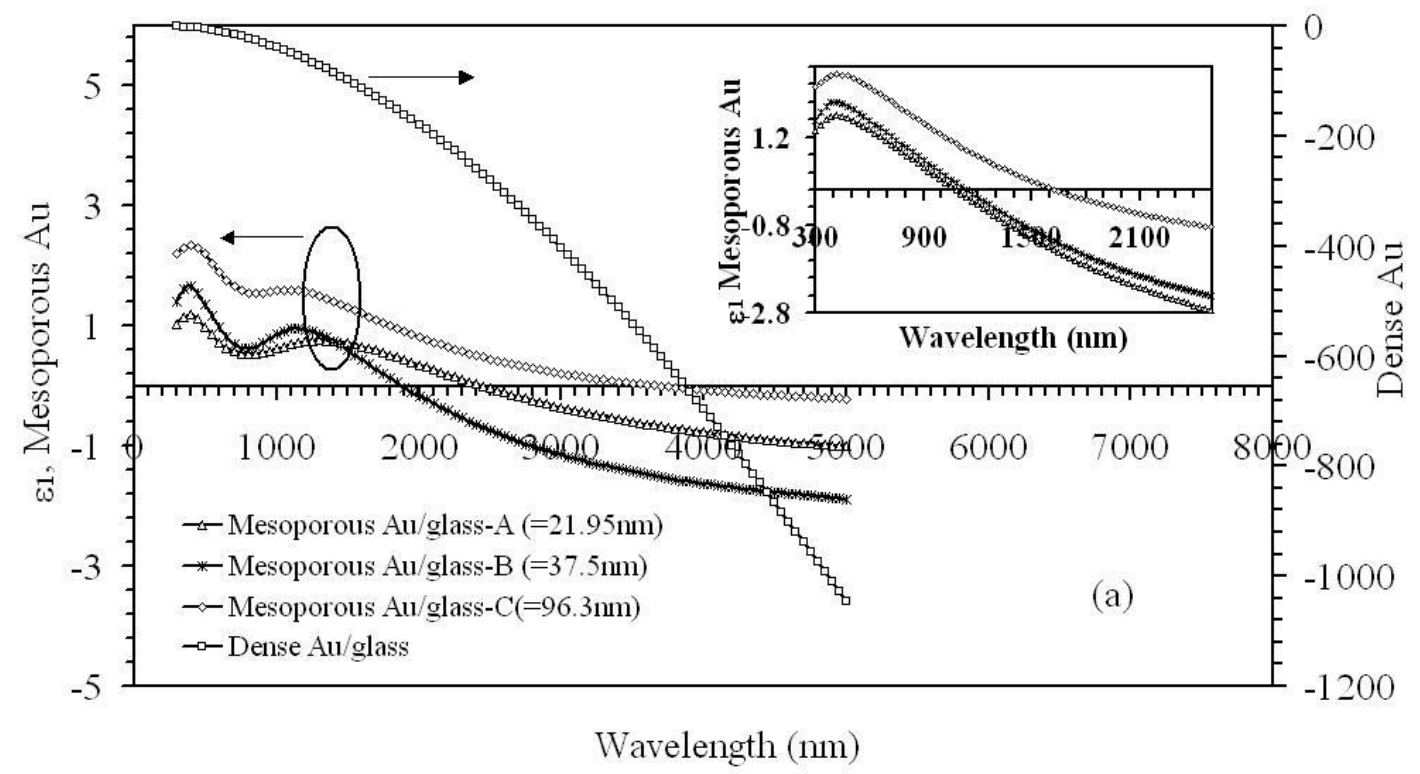




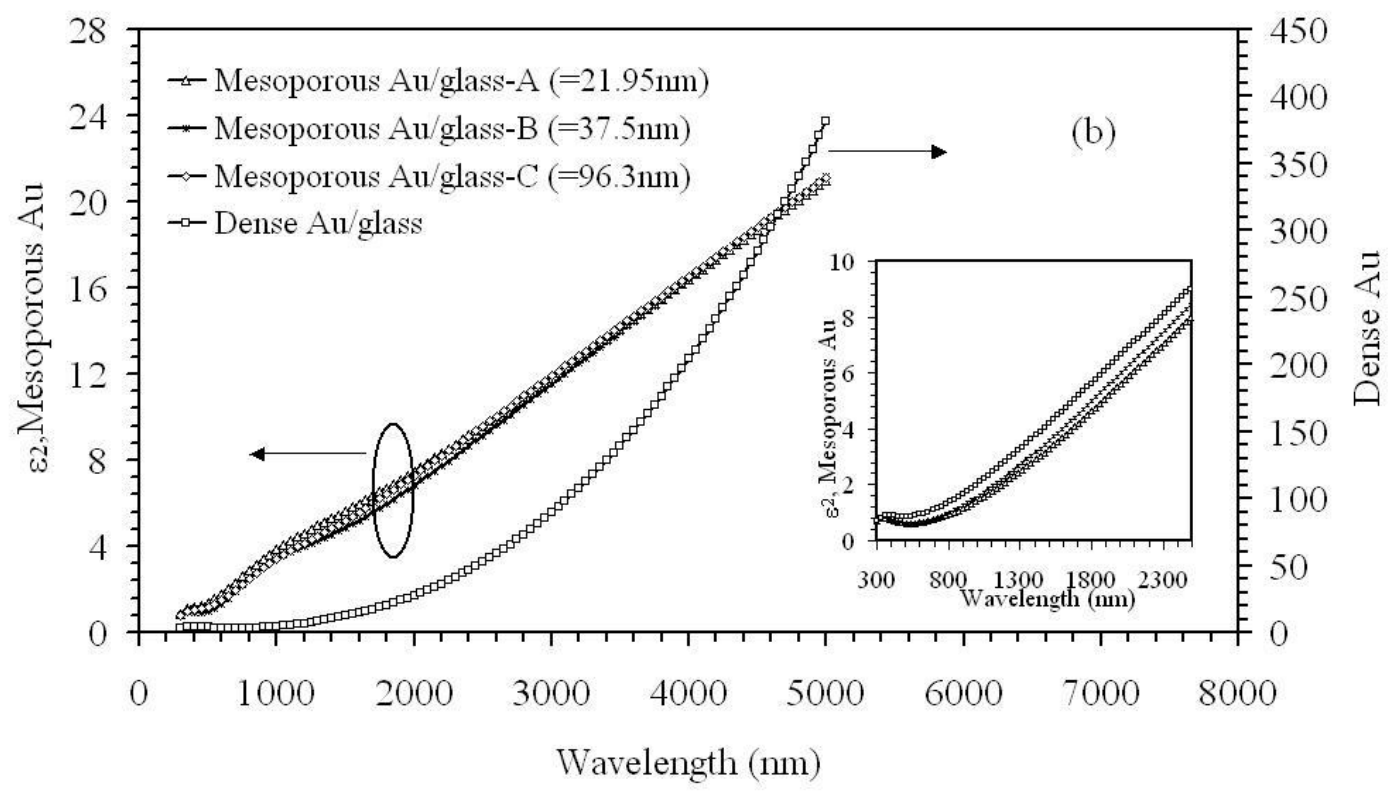

Figure 3. Optical dielectric constants (a) $\varepsilon_{1}$ and (b) $\varepsilon_{2}$ as a function of wavelength for the three mesoporous gold films of thickness $22 \mathrm{~nm}, 37.5 \mathrm{~nm}$, and $96.3 \mathrm{~nm}$ on glass substrate obtained from fitting spectrophotometry data in figure 2 with L-D model. Inset graphs show the optical dielectric constants $\varepsilon_{1}$ and $\varepsilon_{2}$ as a function of wavelength replotted by excluding an NIR oscillator.

\subsection{Thermal emittance in mesoporous gold layers}

The black body thermal emittances of the samples in the main part of figure 2 have been measured on a Windbourn emissometer set for surface temperatures of $100^{\circ} \mathrm{C}$. They are tabulated in table 2 . The results correlate with the observed long wavelength spectra. It is interesting to be able to create such a wide range of emittance by varying thickness and void density in a layer, which still maintains good electrical conductivity. Such an engineering capability using just one material has not previously been demonstrated.

Table 2: Thermal emittance of the samples in figure 2 deduced from the IR reflectance using $\mathrm{E}=1-\mathrm{R}$.

\begin{tabular}{llcl}
\hline \hline Sample & $\begin{array}{c}\text { Thickness } \\
(\mathrm{nm})\end{array}$ & $\begin{array}{c}\text { Reflectance } \\
\left(\mathrm{R}_{\mathrm{IF}}\right)\end{array}$ & Emittance \\
\hline Dense-Au & 10.7 & $0.94 \pm 0.02$ & $0.06 \pm 0.02$ \\
Porous-Au-(A) & 21.95 & $0.13 \pm 0.02$ & $0.87 \pm 0.02$ \\
Porous-Au-(B) & 37.5 & $0.24 \pm 0.01$ & $0.76 \pm 0.01$ \\
Porous-Au-(C) & 96.3 & $0.38 \pm 0.02$ & $0.62 \pm 0.02$ \\
\hline \hline
\end{tabular}

3.5. Void densities, plasma frequencies and effective masses

It is useful to link void densities with plasma frequency and nanostructure. In addition we have recently shown that one can introduce an effective mass for the homogenized plasmonic system, which depends on void density and structure [19]. Two approaches were introduced (i) a fit with the Bruggemann model (high porosity) or Maxwell Garnett model (low porosity) across the complete spectrum and (ii) a fit with a Bergman-Milton model at wavelengths where the Drude response dominates. The results for the samples reported above are in table 3. Void densities agree well from the two approaches and are quite high for a percolating conductor. The relative void densities are hard to compare from fig. 1 due to the two quite distinct nanostructures. While it might appear that the ion-assisted film has more voids, close 
examination shows that the larger features in the other layers also contain nanovoids so that their overall density is lower as found also in table 3. In a previous study [18] by varying pressure during deposition but maintaining a constant mass deposited, void density was altered at the same mass thicknesses. The variation of $f$ with thickness in table 3 indicates that structure continues to evolve with mass deposited at constant pressure. As seen in reference [19] a percolation threshold for very small gold content can mean a high effective mass, but the latter also depends on overall nanostructure which varies as seen in fig 1 . This variation shows up in the models via differences in the Bruggemann parameter $L$ as seen in table 3. Both $L$ and $f$ impact on $m * / m$. The effective bulk plasma frequencies have been successfully modelled by one of equations (5) and (6) [19], with the subscripts referring to the two models in table 3 . The simplest way to tune $\omega_{\mathrm{p}}$ is thus by varying $f$, but care is needed since if structure changes as $f$ changes, $L$ will not remain fixed and the resultant shift in $\omega_{\mathrm{p}}$ will differ from that at fixed $L$. A greater understanding of these issues and hence more precise controlled design of $\omega_{\mathrm{p}}$, must await further studies.

$$
\begin{aligned}
& {\left[\frac{\omega_{\mathrm{p}}}{\omega_{\mathrm{p}(\mathrm{Au})}}\right]_{\mathrm{MG}}^{2}=\left[1-\frac{\mathrm{f}}{1-(1-\mathrm{f}) \mathrm{L}}\right],} \\
& {\left[\frac{\omega_{\mathrm{p}}}{\omega_{\mathrm{p}(\mathrm{Au})}}\right]_{\mathrm{Br}}^{2}=\left[1-\frac{\mathrm{f}}{1-\mathrm{L}}\right]}
\end{aligned}
$$

Table 3: Comparison between effective medium parameters of Maxwell Garnett and Bruggeman with Bergman-Milton models from the fitting experimental data of the samples in

\begin{tabular}{|c|c|c|c|c|c|c|}
\hline \multirow{2}{*}{$\begin{array}{l}\text { Sample and } \\
\text { Thickness }\end{array}$} & \multicolumn{4}{|c|}{ Effective medium parameters } & \multicolumn{2}{|c|}{ Bergman-Milton model } \\
\hline & Model & $\mathrm{L}$ & $\mathrm{f}_{(\text {Void })}$ & $\omega_{\mathrm{p}} / \omega_{\mathrm{p}(\mathrm{Au})}$ & $\mathrm{f}^{\text {Bergman }}$ (Void) & $\mathrm{m}^{*} / \mathrm{m}$ \\
\hline $\begin{array}{l}\text { Porous Au-A } \\
(21.95 \mathrm{~nm})\end{array}$ & $\mathrm{Br}$ & 0.25 & 0.72 & 0.24 & 0.71 & 5.44 \\
\hline $\begin{array}{l}\text { Porous Au-B } \\
(37.5 \mathrm{~nm})\end{array}$ & $\mathrm{Br}$ & 0.15 & 0.80 & 0.25 & 0.79 & 2.90 \\
\hline $\begin{array}{l}\text { Porous Au-C } \\
(96.2 \mathrm{~nm})\end{array}$ & $\mathrm{Br}$ & 0.07 & 0.86 & 0.27 & 0.86 & 1.86 \\
\hline $\begin{array}{l}\text { Porous Au with } \\
\text { Ion assist (36nm) }\end{array}$ & MG & 0.33 & 0.64 & 0.37 & 0.53 & 1.26 \\
\hline
\end{tabular}
figure 2 as given in reference [11]

\section{Absence of surface plasmons}

Despite the strong surface nanostructure evident in the micrographs here and others in reference [16] we can find little evidence for surface plasmon polaritons at the outer surfaces of highly porous samples. If present they would be expected to have a significant impact on optical response. This means in practice that we can have a rigorous metal-like optical response in the presence of strong surface nanostructure, but do not have to be concerned about SPP's. 
We developed an approach to examining if intrinsic SPPs are a significant influence on our optical data on metal films recently [4]. In brief we extract the optical constants of the bare nanostructured metal layer, and then of the same layer over coated with a dielectric whose optical constants had also been measured separately using identical deposition conditions. When SPPs are an influence the measured optical constants of the metal layer apparently shift significantly. This is not of course a real shift but a result of the fact that the SPPs influence is not in the thin film models, and the position of the SPP changes when the neighbouring dielectric changes, because the evanescent fields are altered. Anomalous absorption then occurs $[10,11]$. For metal layers with nanostructure we find significant apparent shift [4], but when we model the stack of $\mathrm{Al}_{2} \mathrm{O}_{3}$ on top of a mesoporous layer we can fit the stack data very well without modifying the metal layer's optical constants. The model for a stack of known thicknesses using original optical constants for each layer by itself is compared to the experimental data for a modelled stack in figure 4 . The fit is clearly excellent and there is no evidence of a shift in $n$ or $k$ in the high porous layer, especially in the long wavelength region where it is expected to be strongest.

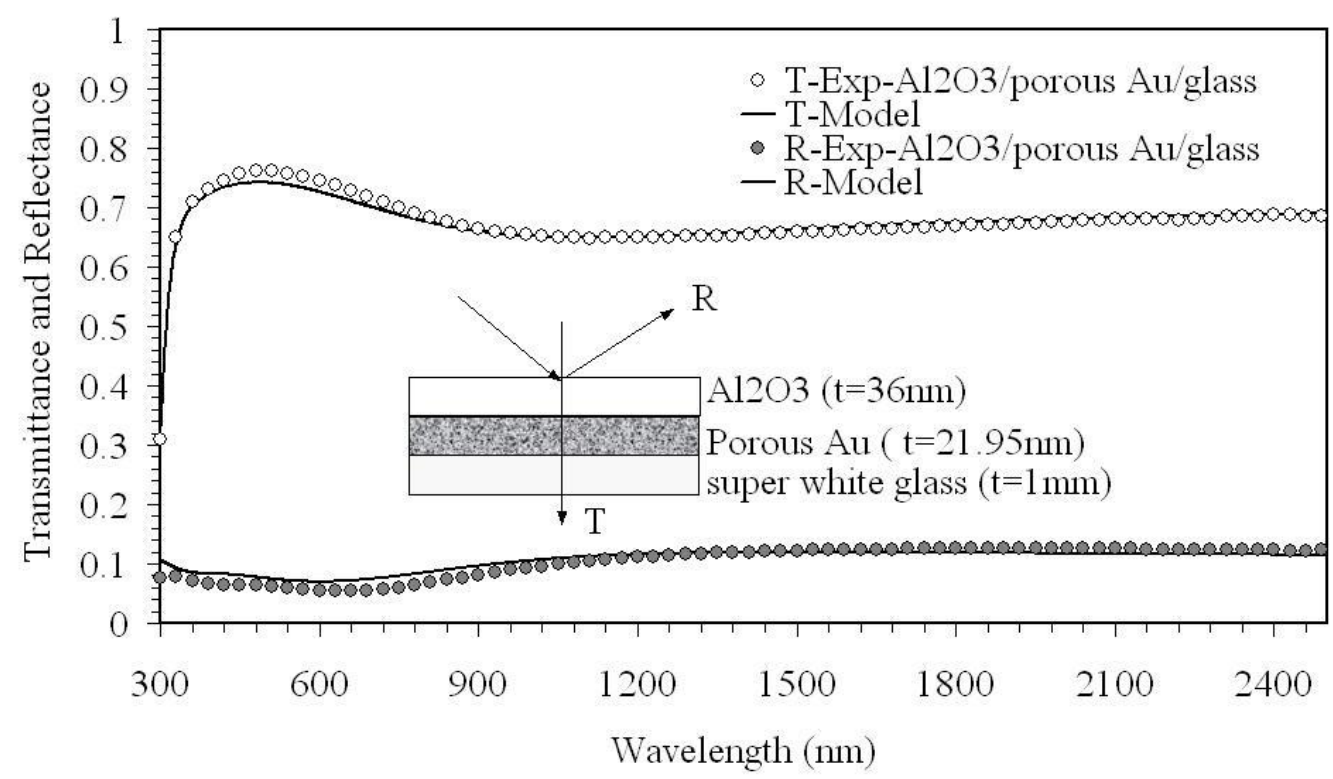

Figure 4. Spectral transmittance and reflectance experimental data of mesoporous gold sample A $(21.95 \mathrm{~nm})$ film coated with thin layer of $\mathrm{Al}_{2} \mathrm{O}_{3}(36 \mathrm{~nm})$ (dotted line) fitted with simple thin films model as shown in the inset graph (sold line).

The quality of fitting with no adjustments to layer parameters in figure 4, especially at longer wavelengths, indicates that interface absorption is absent and hence that surface plasmon polaritons have not been formed in this conductor. Extending this type of analysis to the less porous and more conducting meso-gold films such as the last sample in table 3 and figure $1 \mathrm{~d}$ appears in figure 5. The fit here is not as good as that in fig. 4 if we use individual layer optical constant values. A detailed analysis at long wavelengths for these type of films indicates there is a small amount of interface absorption. (An excellent fit is possible with the addition of a very thin highly absorbing interface layer). Some short range SPPs seem to be forming on this surface and the addition of a dielectric layer then alters their contribution. The effect is however much less than found in overcoated granular metal films [4]. The greater density of gold in these layers has allowed limited generation of short-lived SPPs, but it is still a reasonable first approximation to neglect them. 


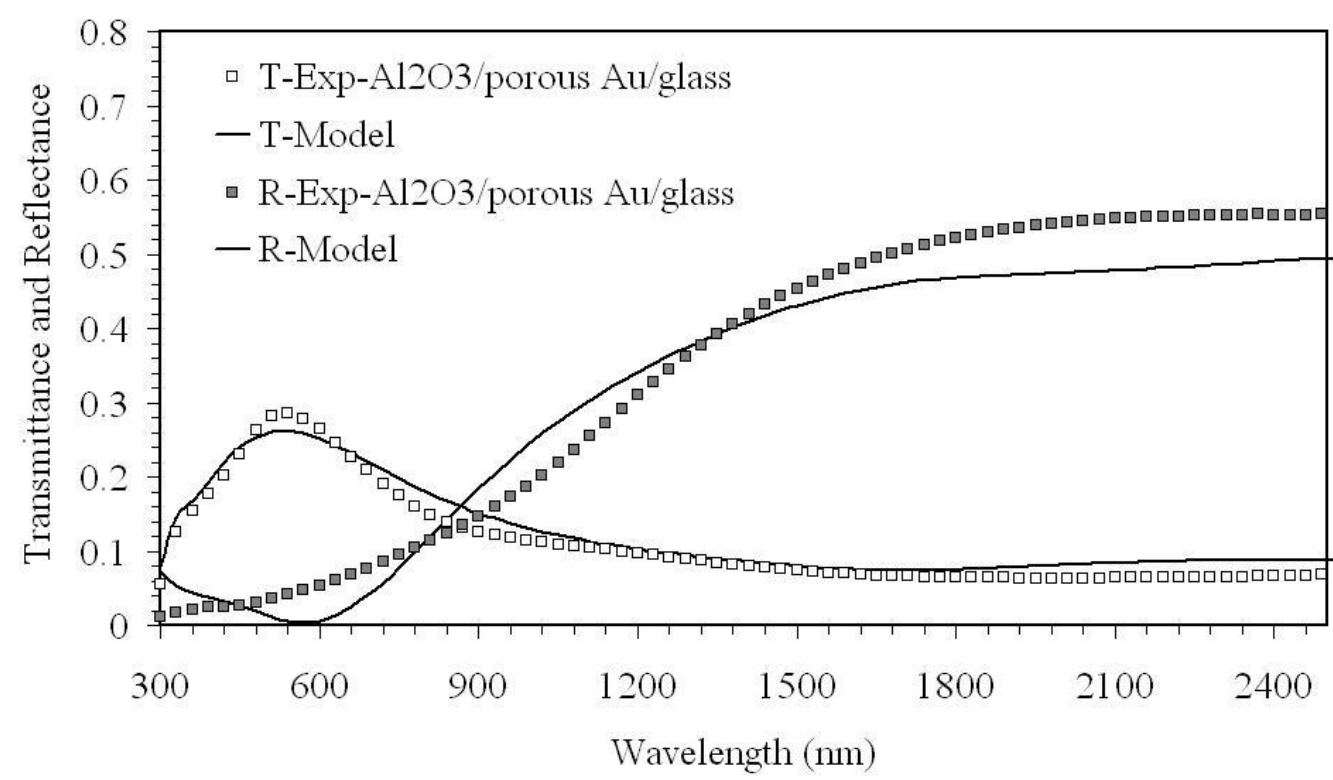

Figure 5. Spectral transmittance and reflectance experimental data of mesoporous gold sample with ion assist film coated with thin layer of $\mathrm{Al}_{2} \mathrm{O}_{3}(36 \mathrm{~nm})$ (dotted line) fitted with simple thin films model as shown in the inset graph of figure 4 (sold line).

In situ spectral studies of thermal stability have been carried out as temperature changes in steps of $20^{\circ} \mathrm{C}$ in vacuum and is then held, backed up by some SEM images. Details will be reported elsewhere but we note here that the mesoporous films are surprisingly stable for thin gold, let alone such a porous structure. It seems the fibrous network may enhance stability. Spectral changes only start to appear above $200^{\circ} \mathrm{C}$. At $300^{\circ} \mathrm{C}$ the structure has collapsed, but not into typical gold islands, but rather gold nano-flakes. The use of these structures in wet environments has also been of interest in terms of whether water is repelled or easily enters the network. Simple electrolytes were able to enter the gold network as reported in references $[17,21,22]$. Water drops on the surface however indicate it is hydrophobic.

\section{Conclusion}

Nanoporous gold containing a network of voids which occupy over $70 \%$ of film volume behave effectively like a uniform Drude metal at NIR frequencies, but becomes plasmonic at much longer wavelengths than in bulk gold. Surface plasmon polaritons do not form on these films despite their surface nanostructure. Less porous gold behaves at NIR wavelengths more obviously like a uniform metal with a higher effective plasma frequency and only a small rise in apparent effective mass. These more conducting layers show slight evidence of formation of SPP's and associated interface absorption, as detected when a dielectric is over-coated onto them. The impact of these SPP's on overall optical response of such layers is quite small. Thus nanoporous gold produced by de-alloying can be used as a "tuneable" uniform plasmonic metal in optical systems but significant changes in optical response due to surface plasmons can be neglected despite the high level of surface nanostructure. Emittance varies widely in these films and can be designed via tailoring void content.

Finally it is worth noting some potential applications of these mesoporous networks, starting with conductors with intermediate thermal emittance in some solar applications and NIR level control in photonics. Others include novel decorative gold coatings, photo-electrochemical and capacitive biosensors, supercapacitors and chemical catalysis. 


\section{Acknowledgments}

We wish to thank Geoff McCredie for help with thin film deposition and MAU for their help with SEM.

\section{References}

[1] Gadenne P, Shubin V A, Sarychev A K, and Shalaev V M, 1999 Phys. Rev. Lett. 82, 337

[2] Sarychev K and Shalaev V M 2000 in Optics of Nanostructured Materials Eds. Markel VA and George T (New York: Wiley)

[3] Seal K, Gresillon S, Aigouy L, Boccara A C, Rivoal J C, Quelin X, Desmarest C, Sarychev A K, Noh H, Genov DA, Yamilov A, Shalaev VM, Ying ZC and Cao H 2005 Phys. Rev. Lett. 94 226101/1-4

[4] Smith G B and Maaroof A I, 2004 Opt. Comm. 242 383-392

[5] Ebbesen T W, Lezec H J, Ghaemi H F, Thio T and Wolff PA 1998 Nature 391 667669

[6] Martin-Moreno L, Garcia-Vidal F J, Lezec H J, Pellerin K M, Thio T, Pendry J B and Ebbeson T W, 2001 Phys. Rev. Lett. 86 1114-1117

[7] Smith G B, Niklasson G A, Svensson J S E M, and C. G. Granqvist 1986 J. Appl. Phys. 59 571-581

[8] Raether H 1988 Surface Plasmons on Smooth and Rough Surfaces and on Gratings (Springer Tracts in Modern Physics 111: Berlin)

[9] Genov DA, Seal K, Sarychev A K, Noh H, Shalaev VM, Ying ZC, Zhang X and Cao H 2006 Appl. Phy. B (Lasers and Optics) B84 205-210

[10] Tachibana Y, Kusunoki K and Ohsaki H 2004 Vacuum 74 555-559

[11] Tachibana Y, Kusunoki K, Watanabe T, Hashimoto K, Kand T and Ohsaki H 2003 Thin Solid Films $\mathbf{4 4 2}$ n 1-2 212-216

[12] Smith G B 2006 Chapter 5 in Frontiers in Optical Technology, Materials and Devices eds. Choudhury P K and Singh O S (Nova Scientific Publishers USA) pp $125-158$

[13] Granqvist C G 1991 Energy Efficient Windows: present and forthcoming technology in Material Science for Solar Energy Conversion Systems (Oxford, UK: Pergamon)

[14] Melville D and Blaikie R 2005 Opt. Exp. 136 2127-2134

[15] Pendry J B 2000 Phys. Rev. Lett. 86 3966-3969

[16] Maaroof A I, Cortie M B and Smith G B 2005 J. Opt. A: Pure Appl. Opt. 7 303-309

[17] Cortie M B, Maaroof A I and Smith G B 2005 Gold Bulletin 38/1 14-22

[18] Smith G B, Maaroof A I and Gentle A 2007 Opt. Comm.s 271 263-268

[19] Smith G B, Gentle A R and Maaroof A I 2007 J. Nanophoto. $1013507-013522$

[20] Maaroof A I, Cortie M B, Gentle A and Smith G B 2007 Phys. B 394 167-170

[21] Mortari A, Maaroof A I, Martin D and Cortie M B 2007 Sensors \& Actuators B, 123 262-268

[22] Yuan L, Liu H K, Maaroof A I, Konstantinov K, Liu J, Cortie M 2007 Journal of New Materials for Electrochemical Systems 10 95-99 